\title{
Welcome to the virus social
}

\author{
Long-known to happen in other realms of the microscopic and macroscopic worlds, social interactions in \\ viruses are increasingly being appreciated and have the potential to influence many processes, including viral \\ pathogenesis, resistance to antiviral immunity, establishment of persistence and even life cycle choice.
}

O ngoing efforts to characterise the virosphere have identified viruses in every environment studied, infecting every life form and even parasitizing other viruses. In addition to this vast viral diversity, variants frequently emerge within populations of a given virus through mutation, deletion, recombination or reassortment. Co-circulation of different viruses in the same areas of the world, sharing hosts and vectors, increases the chances of co-infection and co-transmission - both of which are known to occur, for example, with Chikungunya, Zika and Dengue virus. Virions were traditionally thought to transmit as individual entities, but we now know that groups can be transmitted in vivo as one infectious unit, as aggregates of enveloped or nonenveloped viruses, within exosomes or plasma-membrane-derived vesicles, or by concentrating on the surface of mammalian gut bacteria ${ }^{1}$. Indeed, genetically diverse virions have been shown to initiate infection in a single cell. Some virus-virus interactions have been well characterised, such as superinfection exclusion, whereby a primary viral infection induces resistance to subsequent infections by similar viruses; reassortment, which is for example an important driver of changes in influenza virus host range; or between replicationdefective viruses - such as adeno-associated viruses or hepatitis D virus - with the helper viruses on which they depend. However, despite being widespread in nature, we know surprisingly little about the extent of viral interactions that could alter transmission or the course of infection.

Social interactions were initially described and used to understand animal behaviour, and were soon studied in bacterial and unicellular eukaryote populations. Perhaps because viruses were considered too simple to interact socially, this concept has only recently been adopted in virology. In this issue of Nature Microbiology, Rafael Sanjuan and colleagues show that the ability of a vesicular stomatitis virus to suppress interferon (IFN)-mediated innate immunity is a social altruistic trait that, though costly for the viruses that carry it and produce less progeny in the short term, enables the replication of other members of the viral population that do not repress IFN ( ref. $^{2}$ ). The demonstration that social evolution rules govern viral innate immune evasion and virulence provides a framework for future study of viral social traits. A vibrant, self-denominated sociovirology community is coalescing around the study of these traits ${ }^{3}$ and has organised what promises to be an interesting session on 'The Social Lives of Viruses' at the American Society for Microbiology annual meeting later this month.

The interactions between viruses and their defective viral genomes (DVGs) are also important determinants of infection outcomes, an issue that Marco Vignuzzi and Carolina Lopez discuss in a Review published online later this month in Nature Microbiology (in press). DVGs have been described in most RNA virus populations, and have been associated with immune stimulation, viral attenuation and persistence. Recent advances have shed light on the mechanistic basis of DVG function, including their interference with full-length virus replication and the structural characteristics that make them potent triggers of the innate immune response. Single cell analyses have shown that paramyxovirus DVGs and full-length viruses preferentially infect two different cell populations and lead to different infection outcomes, which is important to promote viral persistence, highlighting the need to consider both the single cell and population-level effects of DVG-virus interactions ${ }^{4}$.

Viruses can interact with those co-infecting the same host, but also communicate with those that will infect subsequently. Some temperate bacteriophages use the arbitrium communication system to determine the number of recent infections in the population by measuring the level of a phage-encoded peptide, and switch to a lysogenic lifestyle to prevent killing off their host when the amount of peptide increases over a certain threshold ${ }^{5}$. Cooperation also allows phage populations to resist bacterial CRISPR-mediated immune defence; initial phage resistance may not be sufficient to overcome the immune response, but creates an immunosuppressive state that enables subsequent infection by other phages in the population ${ }^{6,7}$. Given the ecological importance of bacteriophages in a wide variety of ecosystems, from soil and marine environments to the mammalian gut, it will be interesting to see whether and how virus interactions affect their roles in processes such as biogeochemical cycling and horizontal gene transfer.

Co-infections of viruses and other microorganisms have also been shown to lead to interactions that have major modulatory effects on pathogenesis and host response, as explored in two recent Nature Microbiology articles studying influenzaStreptococcus pneumoniae interactions ${ }^{8,9}$. With growing evidence that microbial interactions are widespread and functionally important, we are happy to have tickets to this social!

Published online: 22 May 2019

https://doi.org/10.1038/s41564-019-0486-6

References

1. Aguilera, E. R. \& Pfeiffer, J. K. Virus Res. 265, 43-46 (2019).

2. Domingo-Calap, P. et al. Nat. Microbiol. https://doi.org/10.1038/ s41564-019-0379-8 (2019).

3. Díaz-Muñoz, S. L., Sanjuán, R. \& West, S. Cell Host Microbe 22, 437-441 (2017).

4. Xu, J. et al. Nat. Commun. 8, 799 (2017).

5. Erez, Z. et al. Nature 541, 488-493 (2017).

6. Borges, A. L. et al. Cell 174, 917-925 (2018).

7. Landsberger, M. et al. Cell 174, 908-916 (2018)

8. David et al. Nat. Microbiol. https://doi.org/10.1038/s41564-0190447-0 (2019).

9. Rowe et al. Nat. Microbiol. https://doi.org/10.1038/s41564-0190443-4 (2019). 